\title{
The End of the Golden Age of Antibiotics?
}

\section{Andrzej Wernicki*}

Faculty of Veterinary Medicine, University of Life Sciences, Lublin,Poland

*Corresponding author: Andrzej Wernicki, Institute of Biological Bases of Animal Diseases, Sub-Department of Veterinary Prevention and Avian Disease, Faculty of Veterinary Medicine, University of Life Sciences, Poland; E-mail: andrzej.wernicki@up.lublin.pl

Citation: Andrzej Wernicki (2013) The End of the Golden Age of Antibiotics? J Vet Sci Anim Husb 1(1): e103. doi: 10.15744/2348-9790.1.e103

Received Date: July 05, 2013 Accepted Date: July 29, 2013 Published Date: August 01, 2013

The discovery of a substance with antibacterial properties was expected to permanently diminish the occurrence of bacterial infections posing a threat to health and life. Since the discovery of penicillin many new groups of antibiotics have been introduced into treatment of disease in people and animals, but their excessive and frequently unjustified use, and most importantly, incorrect dosage, have led to numerous unanticipated problems in contemporary human and veterinary medicine. The most serious of these is the continually increasing number of bacteria resistant to commonly used antibiotics, including drugs of last resort. This phenomenon currently affects all species of microbes, as well as all antibiotics. This situation necessitates strict adherence to the principles of safe and effective antibiotic therapy, regular monitoring of antibiotic resistance in bacteria isolated from animals intended for food production, as well as from pets, wild animals and their living environments, and a search for new ways of fighting bacteria. Strategies should also include efforts aimed at an international ban on the use of antibiotics as growth stimulators in animals, and the development of practices in animal husbandry that reduce the risk of emergence of antibiotic resistance. It is also essential to increase research potential in such areas as genetic improvement of animals aimed at identifying markers associated with enhanced innate resistance to pathogens; the search for new antimicrobial agents; determination of the role of bacteria occurring in animal husbandry in the transmission of antibiotic resistance to bacterial flora in humans and the associated potential risk; and other strategies for preventing and controlling infectious diseases in animals.
Strategies currently being implemented to overcome antibiotic resistance are based on the alternative use of bacteriophages or their lytic enzymes, new vaccines, and bactericidal or bacteriostatic proteins or peptides synthesized by bacteria, plants, invertebrates, vertebrates and mammals. About a thousand of these substances have thus far been described, of which the most important in terms of their potential use in treatment of humans and animals are cysteine-stabilized cationic peptides, also referred to as natural antibiotics.

Another excellent alternative to antibiotics is pro-, pre- and synbiotics, which exert a beneficial influence on the host organism by enhancing the development of health-promoting strains of intestinal bacteria while eliminating pathogenic strains. Another means of significantly reducing the use of antibiotics, by shortening the duration of antibiotic therapy against antibiotic-resistant pathogens, is the use of vitamin D. Research is also being conducted on the potential use of photodynamic therapy and silver nanoparticles.

I was inspired to write these few paragraphs by an article by Alan P. Johnson and Neil Woodford published in the Journal of Medical Microbiology (2013), 62, 499-513., entitled "Global spread of antibiotic resistance: the example of New Delhi metallo- $\beta$-lactamase (NDM)-mediated carbapenem resistance". The information presented by the authors illustrating the speed with which resistance genes can spread all over the world confirms the alarming growth of the problem, which affects public health on a global scale and whose resolution requires international cooperation.

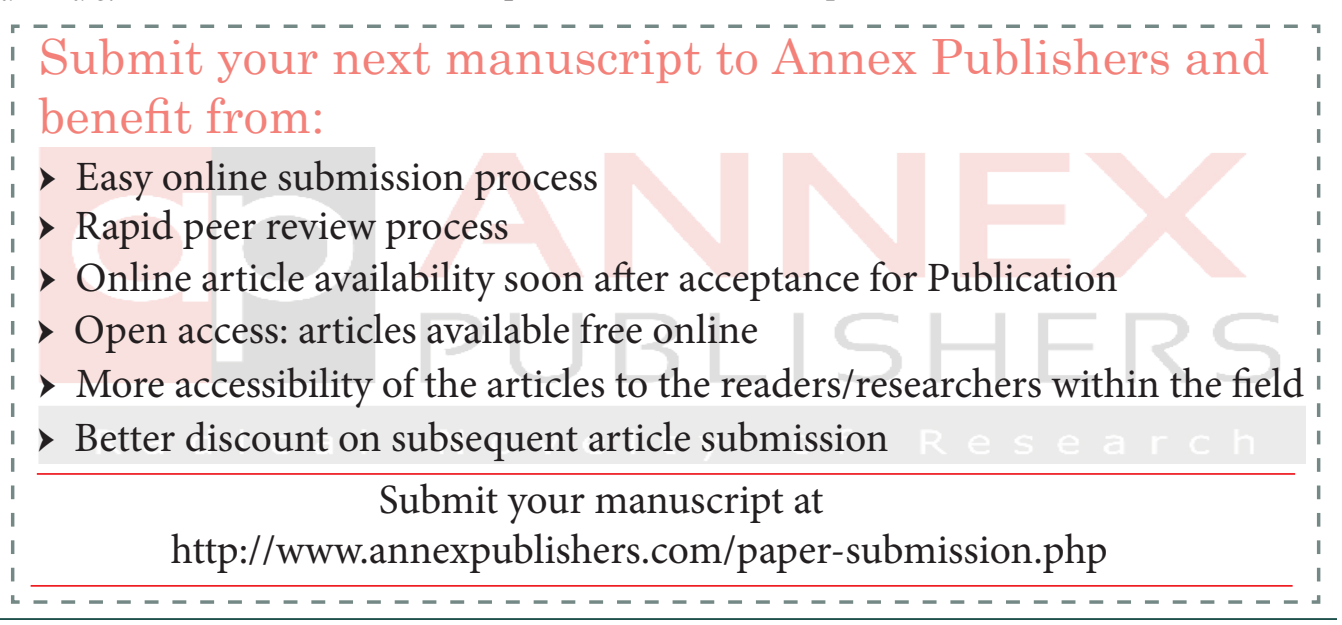

